\title{
Posterior lumbar dynamic stabilization instead of arthrodesis for symptomatic adjacent-segment degenerative stenosis: description of a novel technique
}

\author{
Hazem Mashaly, MD, ${ }^{1,2}$ Erin E. Paschel, PA-C, ${ }^{1}$ Nicolas K. Khattar, MD, ${ }^{1}$ \\ Ezequiel Goldschmidt, MD, PhD, ${ }^{1}$ and Peter C. Gerszten, MD, MPH ${ }^{1}$
}

1Department of Neurological Surgery, University of Pittsburgh School of Medicine, University of Pittsburgh Medical Center Health System, Pittsburgh, Pennsylvania; and 2Department of Neurological Surgery, Ain Shams University, Cairo, Egypt

\begin{abstract}
OBJECTIVE The development of symptomatic adjacent-segment disease (ASD) is a well-recognized consequence of lumbar fusion surgery. Extension of a fusion to a diseased segment may only lead to subsequent adjacent-segment degeneration. The authors report the use of a novel technique that uses dynamic stabilization instead of arthrodesis for the surgical treatment of symptomatic ASD following a prior lumbar instrumented fusion.
\end{abstract}

METHODS A cohort of 28 consecutive patients was evaluated who developed symptomatic stenosis immediately adjacent to a previous lumbar instrumented fusion. All patients had symptoms of neurogenic claudication refractory to nonsurgical treatment and were surgically treated with decompression and dynamic stabilization instead of extending the fusion construct using a posterior lumbar dynamic stabilization system. Preoperative symptoms, visual analog scale (VAS) pain scores, and perioperative complications were recorded. Clinical outcome was gauged by comparing VAS scores prior to surgery and at the time of last follow-up.

RESULTS The mean follow-up duration was 52 months (range 17-94 months). The mean interval from the time of primary fusion surgery to the dynamic stabilization surgery was 40 months (range 10-96 months). The mean patient age was 51 years (range 29-76 years). There were 19 (68\%) men and $9(32 \%)$ women. Twenty-three patients (82\%) presented with low-back pain at time of surgery, whereas 24 patients (86\%) presented with lower-extremity symptoms only. Twenty-four patients (86\%) underwent operations that were performed using single-level dynamic stabilization, 3 patients $(11 \%)$ were treated at 2 levels, and 1 patient underwent 3-level decompression and dynamic stabilization. The most commonly affected and treated level (46\%) was L3 -4 . The mean preoperative VAS pain score was 8 , whereas the mean postoperative score was 3 . No patient required surgery for symptomatic degeneration rostral to the level of dynamic stabilization during the follow-up period.

CONCLUSIONS The use of posterior lumbar dynamic stabilization may offer a valid and safe option for the management of patients who develop ASD rostral to a previously instrumented arthrodesis. The technique may serve as an alternative to multilevel arthrodesis in this patient population. By implanting a dynamic stabilization device instead of an extension of a rigid construct, this might translate into a reduction in the development of yet another level of ASD. http://thejns.org/doi/abs/10.3171/2015.10.FOCUS15413

KEY WORDS adjacent-segment disease; degenerative disc disease; dynamic stabilization; lumbar spinal fusion; motion preservation

$\mathrm{S}$ PINAL arthrodesis has become a common technique in the surgical treatment of symptomatic degenerative disease of the lumbar spine. Posterior instrumented lumbar fusion is a common surgical procedure performed for a large spectrum of clinical indications in the lumbar spine, including degenerative disorders, tumors, infection, and trauma. ${ }^{4}$ Technological advances have resulted in in- creased fusion rates, while decreasing the need for postoperative immobilization and brace therapy. ${ }^{3,13}$ However, there is a growing body of evidence that lumbar fusion may have undesirable long-term effects on the remainder of the spine, particularly on the immediately adjacent motion segments. ${ }^{5,7}$

Although instrumented fusion has been demonstrated

ABBREVIATIONS ASD = adjacent-segment disease; $\mathrm{PET}=$ polyethylene terephthalate; VAS = visual analog scale.

SUBMITTED August 24, 2015. ACCEPTED October 27, 2015.

INCLUDE WHEN CITING DOI: 10.3171/2015.10.FOCUS15413. 
to have satisfactory clinical results, it has been reported to accelerate degenerative changes at adjacent levels. Adjacent-segment disease (ASD) is a term used to describe any abnormal symptomatic change that can develop in the spinal segment adjacent to a solid fusion level, such as disc herniation, lumbar instability, stenosis, or facet joint arthropathy. ${ }^{17}$ Adjacent-segment disease also represents the symptomatic degenerative changes of the adjacent level resulting in clinical presentation of back pain and/or radiculopathy. ${ }^{9}$ This degeneration is typically seen rostral to a fused segment but may also occur caudal to a fusion, especially when the fusion occurs at the L4-5 level. Many studies have reported on an acceleration of the process of degeneration in the spinal segment adjacent to a level of prior arthrodesis that may result in poor clinical outcome and sometimes a need for further surgery. ${ }^{18}$

Multiple dynamic stabilization systems are currently available, and all use a variety of motion-preserving technologies ranging from semirigid rods to ball-and-socket joints. ${ }^{2}$ The use of dynamic stabilization for the treatment of ASD at the rostral end of an instrumented arthrodesis might therefore reduce further progression of the degenerative process by decreasing hypermobility and stresses in adjacent levels. ${ }^{10,22}$ At our institution, the Dynesys Dynamic Stabilization System (Zimmer Spine) has been used for this clinical indication. This study was undertaken to evaluate a series of patients in whom posterior dynamic stabilization was used instead of arthrodesis for symptomatic adjacent-level stenosis of the lumbar spine. Approval for the study was received from the University of Pittsburgh's institutional review board.

\section{Methods}

A retrospective study at a single institution was performed to review and evaluate a cohort of patients who underwent posterior lumbar dynamic stabilization instead of arthrodesis for symptomatic ASD with a history of instrumented lumbar fusion. Patients with degenerative lumbar disc disease were chosen to undergo the procedure if they were candidates for fusion and had symptomatic adjacent-level pathology in which dynamic stabilization was believed to be more appropriate than arthrodesis. The inclusion criteria for patient enrollment were symptomatic (back pain and/or radiculopathy) ASD with a history of instrumented fusion. Conservative treatment must have failed in all patients for at least 6 months. Medical records were reviewed to include demographic data, comorbidities, smoking status, preoperative symptoms, and visual analog scale (VAS) pain scores. Clinical outcome was gauged by comparing VAS scores prior to surgery and at the time of the last follow-up visit. Perioperative data such as treated spinal segment, blood loss, complications, and hospital stay were also analyzed. Preoperative and postoperative imaging studies including radiographs, MR images, CT scans, and provocative discograms were reviewed when available. Furthermore, the imaging studies obtained prior to the primary (fusion) surgery were reviewed to identify whether the levels involved in ASD demonstrated any signs of degeneration prior to the index surgery.

A decompressive laminectomy was performed in all cases such that the neural elements were completely and adequately decompressed prior to placement of the dynamic stabilization instrumentation. The Dynesys Dynamic Stabilization System (Zimmer Spine) was used in all cases (Fig. 1). The Dynesys system is composed of pedicle screws, polyethylene-terephthalate (PET) cords, and polycarbonate urethane spacers. The spacers are placed bilaterally between the pedicle screw heads to withstand compressive loads. The cords are run through the hollow core of the spacers and stabilize the construct by a tensile preload. ${ }^{20}$ The Dynesys system is currently approved by the US FDA as an adjunct to spinal fusion. Its use for this study was in an off-label manner.

Patients are placed prone with care taken to preserve the natural lordosis of the lumbar spine. A standard midline lumbar incision over the spinous processes of the vertebrae is used with a bilateral subperiosteal muscle separation approach. This approach is necessary to properly expose the previously placed instrumentation as well as the new symptomatic adjacent level. All pedicle screws are placed under real-time fluoroscopy to ensure appropriate placement. Correct screw placement is absolutely necessary for optimal functioning of the Dynesys system. A spacer template is used to determine the correct position of the screws. Pedicle screws are placed lateral to the facet joints to avoid facet disruption. A spacer template is used to determine the correct position of the screws relative to one another, to allow for optimal placement of the spacer device. A pedicle probe is used to create a channel for the screw under fluoroscopic guidance. Every attempt is made to use the largest-diameter and longest screw possible according to the patient's anatomy. The pedicle screw is advanced in a lateral-to-medial trajectory until the head of the screw is at the synovium of the joint.

Following pedicle screw placement, a pedicle distance gauge is placed between the screw heads in the center of the holes to measure the appropriate spacer length. The distance (spacer length) is measured with a slight distraction force. The PET cord is then advanced through the first screw with at least $10 \mathrm{~mm}$ of cord remaining outside the screw head. The antitorque instrument is placed onto the screw head. The set-screw driver is applied to the screw

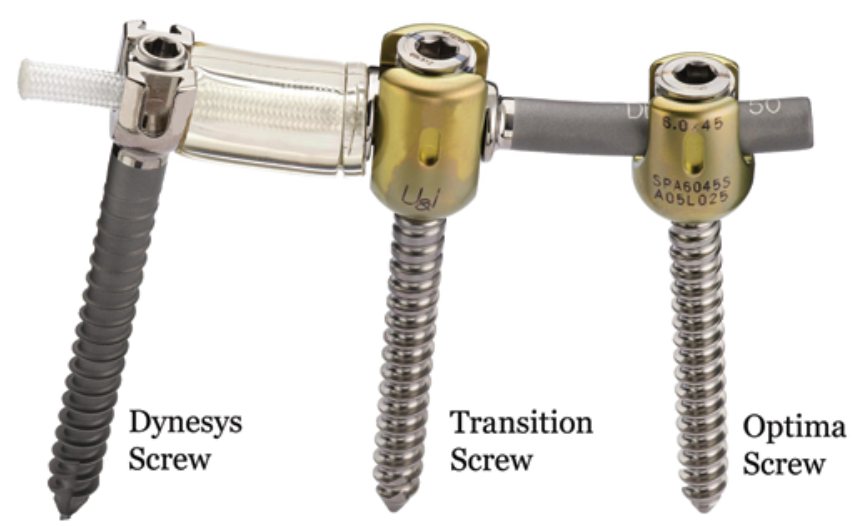

FIG. 1. Photograph of the Dynesys/Transition/Optima (DTO) implant, which is a hybrid construct with a dynamically stabilized segment (on the left) and a rigidly fixated segment (on the right). Image used with permission from Zimmer Spine, Inc. 
head and tightened until the torque-limiting driver snaps. The cord is pushed through the appropriately sized spacer, and the spacer is placed against the first screw head. The cord is then inserted through the second screw. The cord guide is placed on the guide pin and screw, and the cordtensioning instrument is placed on top of the cord guide. Care is taken to keep the cord, spacer, and screws in absolute proper alignment.

The cord-tensioning instrument is used to pull the spacer into proper position. The set screw is inserted into the cord guide using the set-screw starter. The set-screw driver is attached to the torque-limiting driver and then engaged with the set screw. While maintaining tension on the cord, the set screw is tightened until the torquelimiting driver snaps. The procedure is repeated for the contralateral side, and can be repeated for adjacent levels if needed. When the system is fully tensioned the cords are cut, leaving at least $10 \mathrm{~mm}$ of cord extending from the screw heads (Fig. 2).

\section{Results \\ Patient Population}

Between 2007 and 2014, a total of 28 patients who had undergone a prior lumbar fusion underwent posterior lumbar dynamic stabilization using the Dynesys System for symptomatic adjacent-segment degeneration and stenosis. The mean follow-up duration was 52 months (range 17-94 months). Twenty-four patients (86\%) presented with lowback pain at the time of surgery, whereas 25 patients $(89 \%)$ presented with lower-extremity symptoms (17 patients

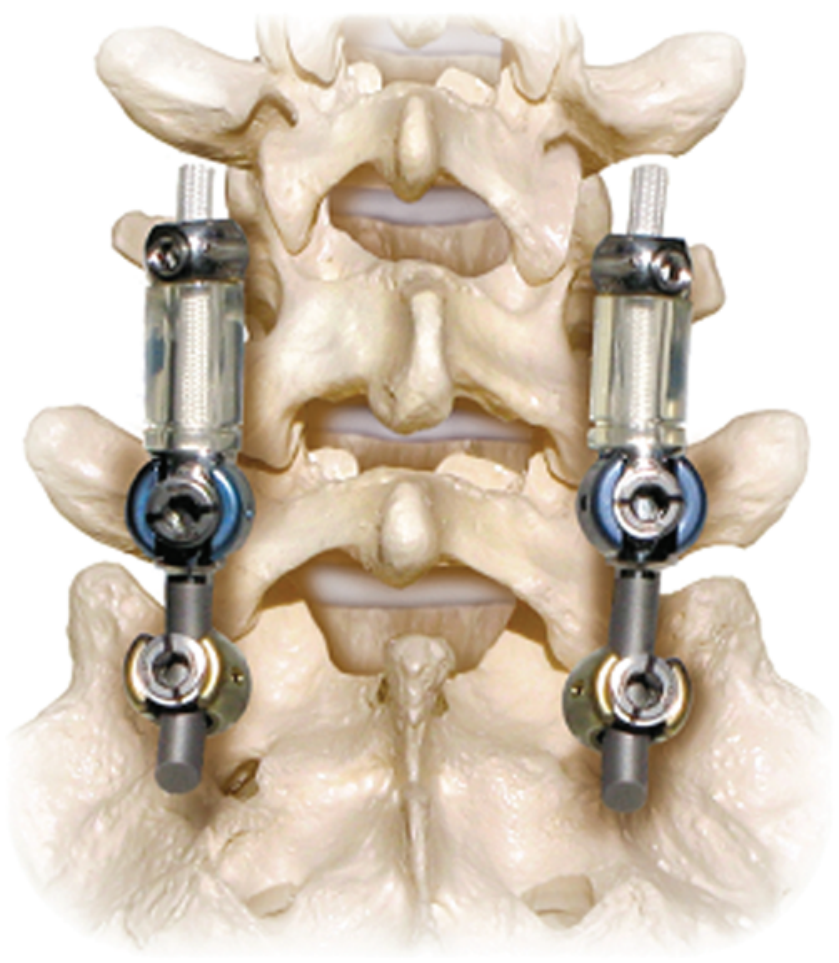

FIG. 2. Illustration of the Dynesys/Transition/Optima (DTO) implant, with a fusion at the L5-S1 level and dynamic stabilization at the L4-5 level. Image used with permission from Zimmer Spine, Inc. with radiculopathy and 8 patients with classic neurogenic claudication). The mean patient age was 51 years (range 29-76 years). There were 19 men (68\%) and 9 women (32\%). Five patients (18\%) had diabetes, 12 patients (43\%) were hypertensive, 2 patients $(7 \%)$ had chronic obstructive pulmonary disease, 2 patients $(7 \%)$ had symptomatic coronary artery disease, and only 1 patient (4\%) had a positive dual-energy x-ray absorptiometry (DEXA) scan for osteoporosis. Ten patients (35\%) were actively smoking at the time of surgery.

\section{Operative Data}

Dynamic stabilization instrumentation was placed immediately following a standard decompressive laminectomy and foraminotomy procedure at the level of symptomatic lumbar stenosis. Twenty-four patients (86\%) underwent operation with single-level dynamic stabilization, 3 patients (11\%) were treated at 2 levels, and 1 patient underwent 3 levels of decompression followed by dynamic stabilization at all 3 levels-for 33 treated levels in 28 patients. The most common instrumented level was L3-4, with 13 patients $(46 \%)$. The L4-5 level was instrumented in 9 patients (32\%), the L2-3 level in 9 patients (32\%), and the L1-2 level in 2 patients (7\%). In addition to the decompressive laminectomy, 4 patients (14\%) underwent concomitant discectomy. In these 4 cases, it was thought that the disk herniation was contributing to the nerve root compression. The average surgical blood loss was $500 \mathrm{ml}$ for the cohort.

\section{Clinical Outcomes}

The mean length of hospital stay was 3 days (range 2-6 days). The mean interval from the time of primary (fusion) surgery to the dynamic stabilization surgery was 40 months (range 10-96 months). For the entire group, the mean preoperative VAS pain score was 8 , and the mean postoperative VAS score was 3. No patient required surgery for symptomatic degeneration rostral to the level of dynamic stabilization during the follow-up period. The preoperative imaging studies at the index fusion surgery were available for 18 patients. Examination of the dynamically instrumented segment showed no evidence of degeneration in 10 patients, whereas in 8 patients there was evidence of degeneration as seen on MRI at the levels that were subsequently treated with decompression and dynamic stabilization (Fig. 3). Surgical complications included intraoperative durotomy that occurred in 4 patients $(14 \%)$. A single patient developed a wound infection that required surgical treatment.

\section{Discussion}

The phenomenon of ASD, referring to accelerated degenerative changes occurring at the ends of the fused spine, has received increasing attention as ever more spinal fusions are performed and long-term follow-up becomes available. ${ }^{5,13,20}$ Adjacent-segment degeneration may occur as a natural part of aging or due to continuation of the initial degenerative process of the spine. However, this phenomenon appears to be accelerated by changes in the range of mobility and stress alteration that follow solid 

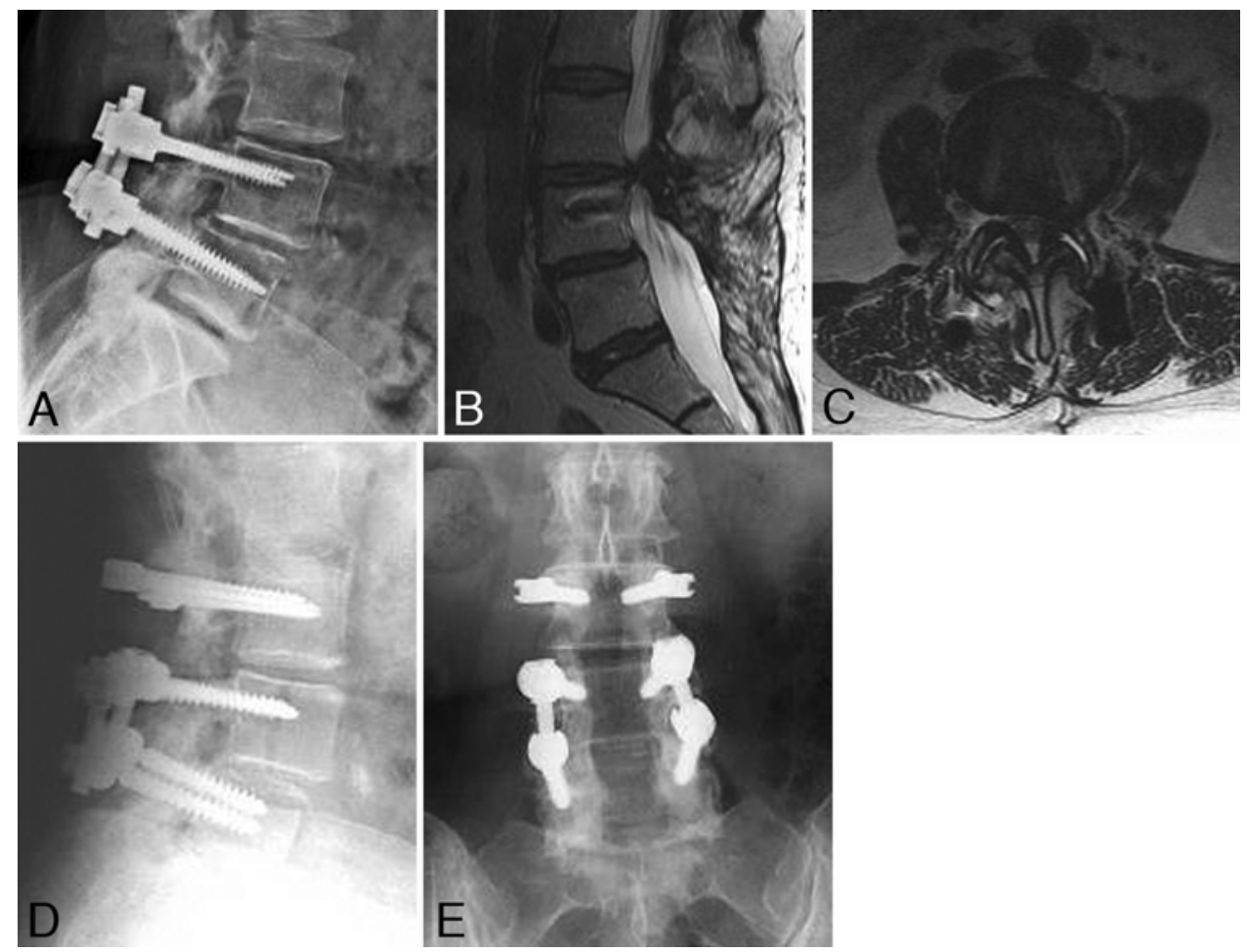

FIG. 3. Case example of a 62-year-old woman who previously underwent an L4-5 pedicle screw fusion, shown on a plain radiograph (A). She presented with symptoms of neurogenic claudication and minimal back pain unresponsive to nonsurgical interventions. A sagittal MRI study (B) revealed severe stenosis at the L3-4 level above her prior fusion associated with bilateral facet disruption, which is seen on axial MRI $(\mathrm{C})$. The disc height was well maintained, and the patient was not believed to be a candidate for an L3-4 fusion. The patient underwent an L3-4 decompressive laminectomy and dynamic stabilization, with resolution of her lower-extremity symptoms. Plain radiographs ( $D$ and $E$ ) demonstrate preservation of the disc height as well as motion at the L3-4 level.

lumbar fusion..$^{16}$ The phenomenon is thought to be due to the altered biomechanics of the fused spine, wherein abnormal forces acting upon the intervertebral discs and facet joints adjacent to the fused segment precipitate the accelerated failure of these stabilizing elements. ${ }^{11}$ Cadaveric studies have described and demonstrated the occurrence of hypermobility and increased stress at the spinal segment adjacent to a solid fusion level. ${ }^{16}$ It has been observed that adjacent-segment degeneration tends to be more common when there was evidence of degeneration in the adjacent level at the time of initial fusion surgery. ${ }^{8}$

Although the time course and prevalence of ASD are not fully known, there is increasing evidence in the spine literature that its effects may be seen soon after fusion surgery, and in as many as $30 \%$ of patients. ${ }^{5,7}$ A large retrospective analysis by Cheh et al. revealed a rate of clinical ASD of $30.3 \%$ and showed that patients who developed adjacent-level disease had significantly worse Oswestry Disability Scale scores that those who did not. These investigators further identified age $>50$ years at time of surgery, increasing length of fusion, and extension of the fusion to L1-3 as significant risk factors for the development of ASD. No significant difference was identified between those undergoing posterior versus circumferential fusion. ${ }^{5}$

Both clinical and laboratory investigations have shown a higher incidence of adjacent-segment degeneration in the levels immediately adjacent to a solid fusion. Lee and
Langrana, among others, showed that there is heightened stress at the facet joints of L3-4 and L4-5 after lumbosacral arthrodesis. Axelsson et al. assessed adjacent segments with the use of radiographic analysis and found hypermobility in the so-called juxta-fused segment.,11,17 In their review, Park et al. showed that by radiological criteria only, the incidence of adjacent-segment degeneration varies anywhere from $8 \%$ to $100 \%$, whereas studies involving symptomatic ASD report an incidence ranging from $5 \%$ to $18 \% .{ }^{17}$ The incidence of adjacent-segment degeneration and ASD following solid lumbar fusion ranged from $14 \%$ to $34 \%$ in yet another publication. 22

In an attempt to address the problem of adjacent-level disease and degeneration, posterior dynamic stabilization devices have been developed. 2,12,13,15,19-21 Posterior dynamic stabilization, in which pedicle screw fixation is coupled with a flexible longitudinal connecting system, presumably allows for the normalization of intersegmental motion. This stands in contrast to traditional fusion surgery, in which the goal is complete and immediate elimination of motion and, ultimately, arthrodesis. By restoring normal motion, mobility is theoretically preserved rather than eliminated, and the forces acting above and below the construct are altered to a lesser extent, reducing the potential undesirable effects of fusion. These devices theoretically avoid an abrupt transfer of stress from a rigid construct to the neighboring segments and thereby potentially dimin- 
ish the risk of adjacent-segment deterioration. ${ }^{23}$ The efficacy of the Dynesys system as a posterior lumbar dynamic stabilization device has been supported by published biomechanical as well as clinical studies. ${ }^{6,10,12,19}$ In their anatomical study, Strube et al. concluded that increasing the fused levels and stiffness of a fusion system would result in compensatory increase of the range of mobility of the adjacent segment, which might lead to or accelerate the degenerative process in the adjacent segment. They also found that using the Dynesys system adjacent to a singlelevel fusion might prevent hypermobility in the adjacent level. ${ }^{22}$ Postoperative MRI and laboratory studies have shown the ability of the Dynesys system to preserve motion in the surgically treated level, while at the same time limiting motion in extension, flexion, and lateral bending. ${ }^{14,22}$

Results from several clinical studies have demonstrated the advantages of a dynamic stabilization procedure in reducing the incidence of adjacent-segment degeneration as compared with fusion surgery. Putzier et al. found that the use of a dynamic stabilization device immediately adjacent to a single-level fusion lowered the incidence of adjacentsegment degeneration at the level immediately adjacent to the treated level to $9.1 \%$, compared with $24.0 \%$ in the isolated fusion group. However, this study also found a $9.1 \%$ incidence of degeneration in the more distant levels. ${ }^{17,18,23}$ In a systematic review of literature, Zhou et al. found that the incidence of adjacent-segment degeneration was statistically lower in patients treated by dynamic stabilization compared with solid fusion. There was a tendency for a lower incidence of ASD following dynamic stabilization. In addition, there was a significantly lower incidence of adjacent-segment degeneration when additional dynamic stabilization systems were applied next to a solid fusion level. ${ }^{23}$

In the current study, a novel technique for the treatment of patients who develop ASD rostral to a previous solid lumbar fusion is reported. Clinical improvement, as measured by changes in VAS pain scores, was consistent with what one would expect in symptomatic patients after a laminectomy and adequate decompression of the neural elements had been achieved. More importantly, with longterm follow-up of 2 to 8 years, no patient in this cohort required a subsequent third lumbar spine arthrodesis surgery due to adjacent-level degeneration rostral to the level of dynamic stabilization. This lack of further surgery is of important clinical significance in this high-risk cohort of patients who already had developed symptomatic adjacent-level pathology requiring at least a second decompressive surgery and stabilization in all cases.

\section{Conclusions}

Posterior dynamic stabilization was used instead of extending a solid fusion on the basis of a growing body of evidence that demonstrates that posterior solid lumbar fusion may accelerate or negatively affect the process of adjacent-segment degeneration and disease. By implanting a dynamic stabilization device instead of an extension of a rigid construct, this might translate into a reduction in the development of yet another ASD. Increasing the number of already fused lumbar segments would accelerate adjacent-segment degeneration. Importantly, the technique was associated with a very low rate of complications. The use of posterior lumbar dynamic stabilization may offer a valid and safe option for the management of patients who develop ASD rostral to a previous instrumented arthrodesis, thus preventing subsequent spinal arthrodesis.

\section{References}

1. Axelsson P, Johnsson R, Strömqvist B, Arvidsson M, Herrlin $\mathrm{K}$ : Posterolateral lumbar fusion. Outcome of 71 consecutive operations after 4 (2-7) years. Acta Orthop Scand 65:309314, 1994

2. Bono CM, Kadaba M, Vaccaro AR: Posterior pedicle fixation-based dynamic stabilization devices for the treatment of degenerative diseases of the lumbar spine. J Spinal Disord Tech 22:376-383, 2009

3. Bono CM, Lee CK: Critical analysis of trends in fusion for degenerative disc disease over the past 20 years: influence of technique on fusion rate and clinical outcome. Spine (Phila Pa 1976) 29:455-463, Z5, 2004

4. Bydon M, Xu R, Santiago-Dieppa D, Macki M, Sciubba DM, Wolinsky JP, et al: Adjacent-segment disease in 511 cases of posterolateral instrumented lumbar arthrodesis: floating fusion versus distal construct including the sacrum. J Neurosurg Spine 20:380-386, 2014

5. Cheh G, Bridwell KH, Lenke LG, Buchowski JM, Daubs MD, Kim Y, et al: Adjacent segment disease following lumbar/thoracolumbar fusion with pedicle screw instrumentation: a minimum 5-year follow-up. Spine (Phila Pa 1976) 32:2253-2257, 2007

6. Cheng BC, Gordon J, Cheng J, Welch WC: Immediate biomechanical effects of lumbar posterior dynamic stabilization above a circumferential fusion. Spine (Phila Pa 1976) 32:2551-2557, 2007

7. Chou WY, Hsu CJ, Chang WN, Wong CY: Adjacent segment degeneration after lumbar spinal posterolateral fusion with instrumentation in elderly patients. Arch Orthop Trauma Surg 122:39-43, 2002

8. Etebar S, Cahill DW: Risk factors for adjacent-segment failure following lumbar fixation with rigid instrumentation for degenerative instability. J Neurosurg 90 (2 Suppl):163-169, 1999

9. Helgeson MD, Bevevino AJ, Hilibrand AS: Update on the evidence for adjacent segment degeneration and disease. Spine J 13:342-351, 2013

10. Kim $\mathrm{CH}$, Chung $\mathrm{CK}$, Jahng TA: Comparisons of outcomes after single or multilevel dynamic stabilization: effects on adjacent segment. J Spinal Disord Tech 24:60-67, 2011

11. Lee CK, Langrana NA: Lumbosacral spinal fusion. A biomechanical study. Spine (Phila Pa 1976) 9:574-581, 1984

12. Lee SE, Park SB, Jahng TA, Chung CK, Kim HJ: Clinical experience of the dynamic stabilization system for the degenerative spine disease. J Korean Neurosurg Soc 43:221-226, 2008

13. Maserati MB, Tormenti MJ, Panczykowski DM, Bonfield CM, Gerszten PC: The use of a hybrid dynamic stabilization and fusion system in the lumbar spine: preliminary experience. Neurosurg Focus 28(6):E2, 2010

14. Niosi CA, Zhu QA, Wilson DC, Keynan O, Wilson DR, Oxland TR: Biomechanical characterization of the three-dimensional kinematic behaviour of the Dynesys dynamic stabilization system: an in vitro study. Eur Spine J 15:913-922, 2006

15. Nockels RP: Dynamic stabilization in the surgical management of painful lumbar spinal disorders. Spine (Phila Pa 1976) 30 (16 Suppl):S68-S72, 2005 
16. Okuda S, Iwasaki M, Miyauchi A, Aono H, Morita M, Yamamoto T: Risk factors for adjacent segment degeneration after PLIF. Spine (Phila Pa 1976) 29:1535-1540, 2004

17. Park P, Garton HJ, Gala VC, Hoff JT, McGillicuddy JE: Adjacent segment disease after lumbar or lumbosacral fusion: review of the literature. Spine (Phila Pa 1976) 29:19381944, 2004

18. Putzier M, Hoff E, Tohtz S, Gross C, Perka C, Strube P: Dynamic stabilization adjacent to single-level fusion: part II. No clinical benefit for asymptomatic, initially degenerated adjacent segments after 6 years follow-up. Eur Spine J 19:2181-2189, 2010

19. Schaeren S, Broger I, Jeanneret B: Minimum four-year follow-up of spinal stenosis with degenerative spondylolisthesis treated with decompression and dynamic stabilization. Spine (Phila Pa 1976) 33:E636-E642, 2008

20. Schmoelz W, Huber JF, Nydegger T, Dipl-Ing, Claes L, Wilke HJ: Dynamic stabilization of the lumbar spine and its effects on adjacent segments: an in vitro experiment. J Spinal Disord Tech 16:418-423, 2003

21. Schnake KJ, Schaeren S, Jeanneret B: Dynamic stabilization in addition to decompression for lumbar spinal stenosis with degenerative spondylolisthesis. Spine (Phila Pa 1976) 31:442-449, 2006

22. Strube P, Tohtz S, Hoff E, Gross C, Perka C, Putzier M:
Dynamic stabilization adjacent to single-level fusion: part I. Biomechanical effects on lumbar spinal motion. Eur Spine J 19:2171-2180, 2010

23. Zhou ZJ, Xia P, Zhao X, Fang XQ, Zhao FD, Fan SW: Can posterior dynamic stabilization reduce the risk of adjacent segment deterioration? Turk Neurosurg 23:579-589, 2013

\section{Disclosures}

Dr. Gerszten is a consultant for Zimmer Spine.

\section{Author Contributions}

Conception and design: Gerszten, Mashaly. Acquisition of data: Gerszten, Mashaly, Paschel, Khattar. Analysis and interpretation of data: all authors. Drafting the article: Gerszten, Mashaly. Critically revising the article: all authors. Reviewed submitted version of manuscript: all authors. Approved the final version of the manuscript on behalf of all authors: Gerszten. Study supervision: Gerszten.

\section{Correspondence}

Peter C. Gerszten, Department of Neurological Surgery, Presbyterian University Hospital, 200 Lothrop St., Ste. B-400, Pittsburgh, PA 15213.email: gersztenpc@upmc.edu. 